\title{
Wearable Neuroimaging: Combining and Contrasting Magnetoencephalography and Electroencephalography
}

Elena Boto ${ }^{1 *}$, Zelekha A. Seedat ${ }^{1}$, Niall Holmes ${ }^{1}$, James Leggett ${ }^{1}$, Ryan M. Hill ${ }^{1}$, Gillian Roberts ${ }^{1}$, Vishal Shah ${ }^{2}$, T. Mark Fromhold ${ }^{3}$, Karen J. Mullinger ${ }^{1,4}$, Tim M. Tierney ${ }^{5}$, Gareth R. Barnes ${ }^{5}$, Richard Bowtell ${ }^{1}$ and Matthew J. Brookes ${ }^{1}$

${ }^{1}$ Sir Peter Mansfield Imaging Centre, School of Physics and Astronomy, University of Nottingham, University Park, Nottingham, NG7 2RD, United Kingdom

${ }^{2}$ QuSpin Inc. 331 South 104 ${ }^{\text {th }}$ Street, Suite 130, Louisville, Colorado, 80027, USA

${ }^{3}$ School of Physics and Astronomy, University of Nottingham, University Park, Nottingham, NG7 2RD, United Kingdom

${ }^{4}$ Centre for Human Brain Health, School of Psychology, University of Birmingham, Edgbaston, Birmingham, B15 2 TT United Kingdom

${ }^{5}$ Wellcome Centre for Human Neuroimaging, UCL Institute of Neurology, University College London, 12 Queen Square, London, WC1N 3BG, United Kingdom

\section{${ }^{*}$ Correspondence to:}

Dr. Elena Boto,

Sir Peter Mansfield Imaging Centre,

School of Physics and Astronomy,

University of Nottingham,

University Park,

Nottingham NG7 2RD

E-mail: elena.boto@nottingham.ac.uk

Pages: 23

Words: 10,252

Figures: 8

Running title: Wearable functional imaging using EEG/MEG 


\begin{abstract}
One of the most severe limitations of functional neuroimaging techniques, such as magnetoencephalography (MEG), is that participants must maintain a fixed head position during data acquisition. This imposes restrictions on the characteristics of the experimental cohorts that can be scanned and the experimental questions that can be addressed. For these reasons, the use of 'wearable' neuroimaging, in which participants can move freely during scanning, is attractive. The most successful example of wearable neuroimaging is electroencephalography (EEG), which employs lightweight and flexible instrumentation that makes it usable in almost any experimental setting. However, EEG has major technical limitations compared to MEG, and therefore the development of wearable MEG, or hybrid MEG/EEG systems, is a compelling prospect. In this paper, we combine and compare EEG and MEG measurements, the latter made using a new generation of optically-pumped magnetometers (OPMs). We show that this new "second" generation commercial OPMs, can be mounted on the scalp in an 'EEG-like' cap, enabling the acquisition of high fidelity electrophysiological measurements. We show that these sensors can be used in conjunction with conventional EEG electrodes, offering the potential for the development of hybrid MEG/EEG systems. We compare concurrently measured signals, showing that, whilst both modalities offer high quality data in stationary subjects, OPM-MEG measurements are less sensitive to artefacts produced when subjects move. Finally, we show using simulations that OPM-MEG offers a fundamentally better spatial specificity than EEG. The demonstrated technology holds the potential to revolutionise the utility of functional brain imaging, exploiting the flexibility of wearable systems to facilitate hitherto impractical experimental paradigms.
\end{abstract}

\title{
KEYWORDS
}

Magnetoencephalography; MEG; Electroencephalography; EEG; Optically-pumped magnetometers; Wearable neuroimaging

\section{HIGHLIGHTS}

- Introduction of $2^{\text {nd }}$ generation, smaller, lighter and highly sensitive OPMs for MEG

- First demonstration of simultaneous EEG and OPM-MEG measurement

- OPM-MEG shown to be less sensitive to artefacts when subjects are allowed to move

- OPM-MEG shown to exhibit fundamentally better theoretical limit on spatial resolution 


\section{INTRODUCTION}

Methods such as functional magnetic resonance imaging (fMRI) and magnetoencephalography (MEG) enable the non-invasive characterisation of human brain function, providing unique insights into the neural substrates that support cognition. However, a significant limitation is that most functional neuroimaging methods require participants to undertake tasks whilst remaining stationary inside cumbersome (and sometimes noisy) scanners. These unnatural surroundings limit the scope of the experiments that can be undertaken: for example, it is hard to exploit environments in which a subject can be fully 'immersed' (e.g. virtual reality); experiments involving realistic social interaction are challenging; studies of spatial navigation, are limited; studying patient groups who exhibit uncontrolled movement (e.g. Parkinson's disease) is extremely challenging. Finally, scanning children using instruments optimised for use in adults is difficult. It follows that the ability to capture brain function using a 'wearable' scanning device, which can be adapted to fit any subject head shape and enable free movement during recording is of significant importance.

Perhaps the best extant example of wearable brain imaging is electroencephalography (EEG), which measures changing electrical potentials at the scalp surface evoked by neural current flow (Berger, 1929). Since EEG only requires small passive electrodes to be placed in electrical contact with the scalp, systems can be integrated into a flexible cap, which is lightweight, and adaptable to any head size. Subjects can therefore move whilst being scanned, making EEG applicable to almost any subject cohort, and any experiment where participants need to move freely. However, EEG also has significant limitations: firstly, the inhomogeneous conductivity profile of the head means that electrical potentials are reduced in amplitude and spatially distorted on the scalp surface (Cooper et al., 1965; DeLucchi et al., 1962). This would not be a problem if such distortions could be taken into account in data processing: however, variations in the shape and thickness of the skull, and changes in conductivity, make these spatial distortions hard to model. These factors place a practical limit on the spatial resolution of EEG (Baillet, 2017). Secondly, previous studies (e.g. Claus et al., (2012); Muthukumaraswamy, (2013)) have shown that EEG is susceptible to artefacts generated by electrical activity in the muscles. This is particularly problematic if paradigms allow (or even encourage) head movement, since EEG data are likely to be impaired by artefacts from muscles in the neck or head. Consequently, a means to supplement EEG with a wearable technique offering better spatial resolution and lower sensitivity to muscle artefacts could offer marked advantages.

MEG (Cohen, 1972; Hämäläinen et al., 1993) measures brain electrophysiology via assessment of extra-cranial magnetic fields, generated by the same neural currents that underlie the EEG signal. Because magnetic fields are less strongly affected by conductivity changes across the head (Baillet, 2017), the MEG forward problem is easier to solve and consequently MEG offers better spatial resolution than EEG. Furthermore, MEG and EEG scalp topographies are orthogonal, and whereas MEG is most sensitive to tangentially-oriented current sources, EEG is most sensitive to radial sources. This means that MEG and EEG are complementary, and a number of studies (Babiloni et al., 2004, 2001; Baillet et al., 1999; Fuchs et al., 1998; Huang et al., 2007; Liu et al., 2002; Molins et al., 2008; Sharon et al., 2007) have exploited this characteristic to demonstrate that the combined use of EEG and MEG offers significant advantages in terms of spatial specificity in source reconstructions compared to using either method alone. However, the magnetic fields generated by the brain are small ( 100 fT) and to achieve sufficient sensitivity to measure them, conventional MEG systems employ superconducting sensors (called superconducting quantum interference devices - SQUIDs) that must be housed inside a liquid helium dewar. This means that conventional MEG systems are large and expensive, and the cryogenically-cooled sensors must be fixed in position (at a reasonable distance from the scalp) within a static helmet. Consequently, MEG systems are 'one-size-fits-all' and subjects must remain still during measurements to avoid loss of data quality. So, whilst 
both MEG and concurrent M/EEG offer technical advantages in terms of spatial precision, current MEG implementations lack the versatility to adapt to different head shapes and exploit naturalistic paradigms. Consequently, practical access to challenging participant cohorts (e.g. infants) is considerably diminished compared to EEG.

Recent studies have begun to describe a new generation of MEG systems (Borna et al., 2017; Boto et al., 2018; livanainen et al., 2019a; Kamada et al., 2015; Kim et al., 2014) which use optically-pumped magnetometers (OPMs) to measure the MEG signal. OPMs rely on the quantum properties of alkali atoms, alongside optical pumping, to make precise measurements of small magnetic fields. Recent work has seen dramatic increases in the sensitivity of these sensors, and a marked reduction in their size (Knappe et al., 2016; Osborne et al., 2018; Sheng et al., 2017). OPMs also benefit from their heavily diminished requirement of thermal insulation compared to SQUIDs: only a few millimetres of insulation is required for the OPM to operate with its external housing at approximately body temperature, compared with several centimetres of fibreglass and a vacuum for SQUID systems. It is these simultaneous reductions in size and sensor-to-head distance which permit OPMs to be mounted on the scalp, allowing adaptation to any head size. With scalpmounting, the sensing volume is positioned only millimetres from the scalp surface and the measured magnetic field is consequently higher compared to superconducting sensors (which are located further away), providing increased sensitivity (Boto et al., 2017, 2016; livanainen et al., 2017). Most significantly, assuming that background magnetic field is close to zero, the subject can move their head freely during data acquisition (Boto et al., 2018; Holmes et al., 2018). A MEG system constructed using OPMs therefore offers an exciting prospect of the technical advantages of MEG, and a practicality that begins to rival that of EEG. Furthermore, it is possible to conceive of a hybrid wearable M/EEG device with OPMs mounted in a cap alongside EEG electrodes.

Whilst the promise of OPM-MEG is exciting, the commercial sensors used in recent implementations have been much larger than EEG electrodes. Consequently, whilst MEG scanning of a moving subject has been achieved, it required sensor-mounting in a rigid 3D-printed helmet, which does not easily allow implementation of simultaneous MEG and EEG measurements. In this paper, we will describe the first MEG experiments undertaken with $2^{\text {nd }}$ generation (Gen-2) commercially-available OPMs. These new devices are much smaller than the original OPMs, enabling mounting on flexible caps alongside EEG electrodes. In addition, the cabling of the new sensors is lighter than in the previous implementations, reducing the weight and bulk of an array, thus better supporting natural movement. We will show that Gen-2 OPMs are a viable means to make high-fidelity MEG measurements. Following this, we aim to demonstrate that OPM-MEG and EEG can be used concurrently, enabling simultaneous measurements of scalp-based electric potential and magnetic field, thus paving the way for a hybrid wearable imaging device exploiting the complementarity of MEG and EEG. Finally, we will contrast the performance of EEG and OPM-MEG, assessing both the artefacts generated by head movements, and (in simulation) the theoretical limits of spatial specificity. 


\section{METHODS}

All experiments were approved by the University of Nottingham Medical School Research Ethics Committee and all participants gave written informed consent to take part in the study. All data and code for data processing are available on request from the corresponding author.

\subsection{System overview and Gen-2 OPMs}

A schematic diagram of our system is shown in Figure 1a. For OPM-MEG, the system comprised two Gen-2 commercial OPMs (QuSpin Inc. Colorado, USA) mounted on the head surface. Each sensor is a self-contained unit incorporating a glass cell containing a ${ }^{87} \mathrm{Rb}$ gas, a $795-\mathrm{nm}$ laser and associated optics for optical pumping, a heater to enable operation in the spin-exchange relaxation-free regime (Allred et al., 2002), a photodetector to measure light transmission through the cell, and 3 on-board electromagnetic coils for control of the field inside the cell. The physical principles underlying these sensors have been described in detail in previous papers (Osborne et al., 2018; Sander et al., 2012; Shah et al., 2007; Tierney et al., 2019). The differences between these Gen-2 OPMs and commercial sensors used in previous MEG studies (Boto et al., 2018, 2017; Holmes et al., 2018; livanainen et al., 2019a; Tierney et al., 2018) are in their size, weight and performance. A photograph of both Gen-1 and Gen-2 sensors can be shown in Figure 1b. For Gen-2 sensors, the package is $1.24 \times 1.66 \times 2.44 \mathrm{~cm}^{3}$ in volume and weighs $4 \mathrm{~g}$. The theoretical noise floor is $\sim 7-10 \mathrm{fT} / \sqrt{\mathrm{Hz}}$ and the on-board coils can generate (and hence null) fields of up to $200 \mathrm{nT}$ over the cell. The stand-off distance between the sensor casing and the sensitive volume is $6.5 \mathrm{~mm}$ and the operational dynamic range (defined as the range of fields over which the sensor gain is stable to $\pm 5 \%$ ) is $\pm 1.5 \mathrm{nT}$. The sensor is connected to control electronics by a 5 -m cable, however the first $60.96 \mathrm{~cm}(2 \mathrm{ft}$ ) of this cable is made from a light 'flex' cable with weight of $3.3 \mathrm{~g} / \mathrm{m}$, to reduce torque on the head caused by cable weight. The lightweight nature and reduced size of these new OPMs (and their cabling) means that they can be mounted onto a flexible cap; this was achieved using two plastic mounts, which were glued to a swimming cap and worn by the subject (Figure 1c). The subject was able to move their head freely throughout data acquisition (within a range $\pm 10 \mathrm{~cm}$ and \pm 34 degrees (see Holmes et al., (2018)). This offers approximately a full range of head motion, without the subject changing position on the seat.

a

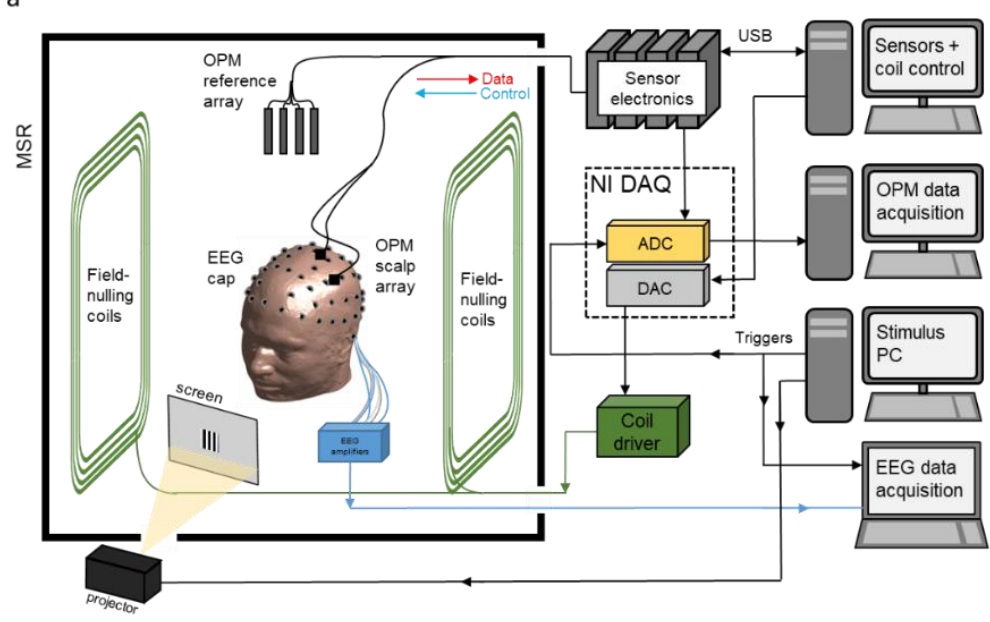

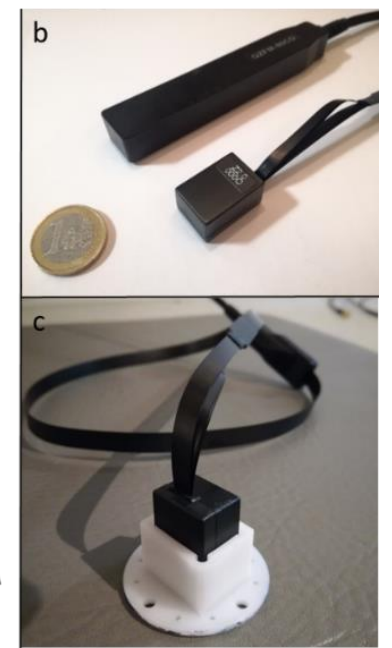

Figure 1: The OPM-MEG system: a) Schematic diagram showing an overview of the complete OPM-MEG system, along with the EEG set-up. b) Photograph showing a comparison of Gen-1 and Gen-2 OPM sensors. c) Photograph of a Gen-2 sensor mounted in a plastic holder. 
Aside from the OPM type, the integrated MEG system was similar to that previously described (Boto et al., 2018). The instrumentation was housed inside a magnetically-shielded room (MSR), and all electronic components were kept outside the room to minimise magnetic interference. A bi-planar coil system (Holmes et al., 2018) was used, in conjunction with a reference array (comprising 4 Gen-1 OPMs placed immediately behind the subject) to null any remnant background field inside the MSR; specifically, reference array measurements enabled calculation of optimised coil currents to remove the static field, and the three dominant field gradients over a central volume. Note that this step is critical to enable subject movement since without it, movement of an OPM (with the head) through the background field will generate magnetic signals larger than the sensor dynamic range (Holmes et al., 2018; livanainen et al., 2019a). The OPM outputs, and control of the background field measurement and coil array was achieved using a National Instruments (NI) digital acquisition system and all data were recorded at a sampling frequency of $1,200 \mathrm{~Hz}$. Stimulus delivery was controlled by an independent computer which also sent trigger signals, via its parallel port, to the digital acquisition system. Visual stimuli were projected through a waveguide in the MSR wall, onto a back-projection screen located approximately 40 $\mathrm{cm}$ in front of the subject. An EEG system was integrated with, and operated independently of the OPMMEG instrumentation (see details below).

\subsection{Motor and visual experiments with Gen-2 OPMs}

Our first experiments were designed to evaluate the viability of Gen-2 OPMs for MEG measurements. We employed two paradigms:

- Motor task: The paradigm involved visual presentation of a centrally-located, maximumcontrast, vertical square-wave grating ( 3 cycles per degree). In a single trial, the grating was presented for $1 \mathrm{~s}$ followed by a 3-s baseline period during which only the fixation cross was shown. During presentation, the participant was instructed to make repeated abductions of their right (dominant) index finger. Previous work has shown that this movement elicits a reduction in beta-band oscillatory amplitude during movement (desynchronisation), and an increase in beta amplitude above baseline (the 'rebound') on movement cessation, both localised to the primary sensorimotor cortices (Pfurtscheller and Lopes Da Silva, 1999). 50 trials were recorded, with blocks of ten trials interspersed with blocks of 'rest' (40 $\mathrm{s}$ in duration) in which the fixation cross was shown and the subject made no movement. A single participant (male, right-handed, age 38 years) took part in the study.

- Visual task: The visual paradigm comprised presentation of a visual stimulus which is known to robustly increase the amplitude of gamma oscillations in primary visual cortex (Hoogenboom et al., 2006; livanainen et al., 2019b). A single trial comprised $1 \mathrm{~s}$ of baseline measurement followed by visual stimulation in the form of an inwardly moving, maximumcontrast circular grating. In $50 \%$ of trials, following either $1.2 \mathrm{~s}$ or $1.4 \mathrm{~s}$ of grating presentation, the speed of motion of the grating increased, and the subject was asked to respond, via button press, as soon as they could following the change in speed. The grating remained on display (moving at the faster rate) for a further $500 \mathrm{~ms}$. In the other $50 \%$ of trials, there was no change in speed and the grating was displayed for $1.6 \mathrm{~s}$ in total. Each trial ended with $5 \mathrm{~s}$ of rest, and a total of 100 trials was used. The subject was given feedback during each trial. A single participant (female, right-handed, age 28 years) took part in the experiment. 
In both experiments, the OPMs were positioned to best capture the neuromagnetic field of interest (Boto et al., 2017). This was achieved using a prior cryogenic-MEG recording in the same subject; briefly, the dipole underlying the beta rebound (in the case of the motor experiment) and the visualgamma response (in the case of the visual experiment) was localised and a forward field was modelled at the scalp surface. This allowed a visual representation of the expected field from each experiment in each subject, and OPMs were positioned, by visual inspection, at the maxima and minima of these modelled fields. Note that at the time of writing only two Gen-2 OPMs were available, and optimised placement of sensors was therefore necessary: in a complete system based around a large number of sensors, this process would be avoided.

The motor and visual experiments were both carried out three times, and we aimed to examine the effect of moving the OPM sensor further from the head. To this end, the plastic holder in which the OPMs were mounted was changed, sequentially placing the outer casing of the OPM at $0 \mathrm{~mm}, 8 \mathrm{~mm}$ and $18 \mathrm{~mm}$ from the scalp surface. To provide a measurement of baseline noise, the same experiments were also undertaken with the sensors mounted (with $0 \mathrm{~mm}$ separation) on a phantom (a polystyrene model head).

For data analysis, the signals from the two OPMs were subtracted to generate an approximation to a planar gradiometer (for magnetometer results, see Supplementary Material); this resulted in a single signal, which was then processed in three different ways:

1) Time-frequency spectrograms: The gradiometer data were frequency filtered into 26 overlapping frequency bands, and a Hilbert transform was used to generate the amplitude envelope of oscillations within each band. These envelope time courses were then segmented into trials and averaged (across trials), independently for each band. Concatenation of the averaged signals in the frequency dimension yielded a time-frequency spectrogram (TFS) showing modulation of oscillatory dynamics.

2) Single-trial responses: To test whether responses in individual trials were visible, we first frequency filtered the data into a band of interest: for the motor task we used the beta (13$20 \mathrm{~Hz}$ ) band, while the gamma $(55-80 \mathrm{~Hz})$ band was used for the visual task. We then plotted the filtered data, with trial markers overlaid, to test for clearly visible responses in each trial.

3) Signal-to-noise ratio: We quantified the absolute signal-to-noise ratio (SNR). For the motor task, we defined the 'signal' as the variance of the post-stimulus beta rebound, which we measured in the $13-20-\mathrm{Hz}$ band and the 1.5-2.4 s time window (relative to trial onset). For the visual task, 'signal' was defined as the variance of the gamma $(55-80 \mathrm{~Hz})$ response in the $0.2 \mathrm{~s}$ to $2.2 \mathrm{~s}$ window relative to onset of the concentric circle pattern. In both cases, 'noise' was defined as the equivalent (frequency-filtered and temporally-windowed) variances, derived from data recorded in a phantom. SNR was measured independently for each trial and averaged. It was also measured independently for each stand-off distance from the scalp.

\subsection{Simultaneous EEG/MEG}

The second set of experiments was designed to examine the viability of simultaneous EEG/OPMMEG; specifically, we aimed to experimentally test: 1) whether high fidelity data could be acquired from EEG and OPM-MEG simultaneously without affecting the efficacy of either modality; and 2) what the effect of head movement on both modalities would be. 
EEG data were acquired using BrainAmp MRplus EEG amplifiers (Brain Products, Munich) with a $1 \mathrm{kHz}$ sampling rate and a frequency range of $0.06-250 \mathrm{~Hz}$ (30-dB roll-off at high frequencies). A MEGcompatible 63-channel EEG cap (EasyCap, Herrsching) comprising $\mathrm{Ag} / \mathrm{AgCl}$ electrodes and copper wires was fitted to the subject, with electrode impedances maintained below $10 \mathrm{k} \Omega$ for the duration of the study (these were checked regularly between recordings). The electrode layout followed the extended international 10-20 system with an additional channel for recording the electrocardiogram (ECG) placed on the subject's back. FCz was used as the reference electrode during acquisition. Stimulus triggers were split such that the trigger signal was simultaneously sent to the EEG and OPM-MEG systems. All data were acquired using BrainVision Recorder software (v1.2, Brain Products, Munich). Having fitted the EEG cap, this was then covered by a second flexible fabric cap, and the OPMs mounted, again in plastic holders, at locations chosen to capture the minimum and maximum of the magnetic field (again assessed visually according to the cryogenic-MEG recording). Data were recorded during two paradigms:

- Motor task: One participant (male, right-handed, age 38 years) undertook the motor experiment (see above for details) six times. In the first two runs, the subject was told to keep as still as possible. In the second two runs the subject was asked to make small, natural movements. In the final two runs, the subject was asked to make large movements, including rotating their head as far as was comfortable, but without changing body position.

- Resting state: We also undertook a series of resting state experiments with a primary aim to assess whether the presence of OPMs would decrease the quality of EEG data, and likewise whether the presence of EEG electrodes would decrease the quality of the OPM-data. A single subject (male aged 39 years) took part in the study. The participant was either wearing: 1) the EEG cap only, 2) the EEG cap with the OPMs on top or 3) the fabric cap with the OPMs only. In each case, we collected ten repeat measurements of four-minute long resting state recordings. In 5 recordings the participant was asked to remain still and the other 5 recordings they were asked to make natural movements.

All EEG data were pre-processed using BrainVision Analyzer2: data were re-referenced to an average of all channels, to provide as clean a reference signal as possible (with minimal neuronal or artefactual signals (Mullinger et al., 2013), and then exported to be analysed using MATLAB (Mathworks Inc., version 2018b). Subsequent data processing was carried out in sensor space.

For the motor task, TFSs and single-trial responses were derived as described above, and we compared OPM-MEG (magnetometer and gradiometer recordings) to the EEG channel with the largest beta-band response (defined using the two stationary runs). To identify the best EEG channel, we first filtered the data from the stationary runs into the beta band $(13-20 \mathrm{~Hz})$, then using a Hilbert transform we generated the amplitude envelope of the signal. The mean amplitude difference between the rebound window (1.5-2.4 s) and the desynchronisation window (0.1-1.0 s) in the task trials (finger abduction), divided by the mean of the envelope in the 0.5-3.5 s window of the rest trials was then used to quantify the magnitude of the beta response. The EEG channel that gave the highest value was selected. This measure was used again to assess the difference in relative beta modulation obtained from the OPM gradiometer and the best EEG electrode. For the resting state data, power spectral density (PSD) measures were obtained, for each of the 4-minute runs, and then averaged over the different conditions (EEG-only, MEG-only, EEG/MEG, static, moving). We then looked for differences in the PSD in $E E G$, caused by the presence of the OPMs, or in MEG caused by the presence of the EEG.

To assess the effect of head movement on OPM-MEG and EEG recordings, we aimed to quantify any measurable muscle artefacts. To this end, we generated an interference metric. Previous evidence has shown that interference from muscle activity tends to manifest at high frequency, and we therefore 
frequency filtered the data into the $75-100 \mathrm{~Hz}$ band. We then used a Hilbert transform to generate the amplitude envelope of this high-frequency signal. Note that in the absence of artefact we would expect the envelope in this band to remain approximately constant. However, if an appreciable muscle artefact was measured, we would expect the envelope to vary significantly throughout the experiment. We therefore used the variance of the envelope, divided by its mean as a normalised measure of interference. This interference metric was measured, in the motor data, for all trials independently, and a trial average and standard deviation across trials derived. In addition, for the resting state data recorded using concurrent OPM-MEG-EEG, we again measured PSD and overlaid spectra, averaged across runs, for EEG and OPM-MEG, recorded with and without movement. We also assessed correlation between the envelope of beta oscillations (temporally down-sampled to $1 \mathrm{~s}$ time resolution) measured using the OPM gradiometer and all EEG channels; this was calculated for stationary and moving runs independently.

\subsection{Spatial resolution}

With only two OPMs, it was impossible to obtain an experimental insight into spatial specificity of OPM-MEG and EEG, and so theoretical limits on spatial resolution were investigated in simulation.

We note that similar simulations have been undertaken previously (Hämäläinen and Lundqvist, 2019) though not in the present context. As noted in the introduction, MEG exhibits better spatial resolution than EEG, however, this is not a fundamental property of the measurement, but rather a data modelling issue originating from the fact that accurately predicting the scalp topography of EEG signals (i.e. solving the EEG forward problem) is challenging. Consequently, inference on the spatial signature of current distribution in the brain based on EEG measurements is compromised. This said, recent novel methods for EEG forward modelling have the potential to improve EEG forward field accuracy, and if comparable accuracy with MEG can be achieved, then ostensibly the spatial resolution of EEG and MEG could be similar. Here, in order to estimate theoretical upper limits on the spatial resolution of EEG and OPM-MEG (for conventional MEG see supplementary material), we made the assumption that both MEG and EEG forward solutions could be solved perfectly.

We simulated two sources in the brain, at locations $\mathbf{r}_{1}$ and $\mathbf{r}_{2}$, separated by a distance $\delta=$ $\left|\mathbf{r}_{1}-\mathbf{r}_{2}\right|$. Both sources were dipolar, with moments $\mathbf{q}_{1}$ and $\mathbf{q}_{2}$, and we assumed that the source strengths were equal. This is shown schematically in Figure 8a. Both sources were simulated at the same orientation with an angle, $\theta$, relative to the radial direction. $\phi_{1}$ and $\phi_{2}$ are the angles between the source orientations and the vector separating the two sources. For simplicity we assumed that the head could be modelled as a sphere of radius $8 \mathrm{~cm}$. A forward solution was derived for EEG using the three concentric sphere model described by Zhang (1995), with brain/skull/scalp conductance's of 0.33/0.0044/0.33 S/m and relative radii $0.87 / 0.92 / 1$. For MEG we assumed the analytical formulation of the forward solution first described by Sarvas (1987). For both MEG and EEG we simulated 800 measurement points over the sphere surface. (Note that this simulation is not intended to represent a real system; rather we intended to probe the basic topological properties of the electric potential and magnetic field.) We assumed that sensors were uniformly spaced: EEG sensors were at the scalp surface and the sensitive volumes of the OPMs were sited $6 \mathrm{~mm}$ above the scalp.

To estimate spatial resolution for both modalities, we took a theoretical approach, using a beamformer. If we let $\mathbf{l}_{1}$ and $\mathbf{l}_{2}$ be the forward solution vectors for dipoles 1 and 2 , respectively, and 
then assume that there is no error in modelling the forward solution, for a two-source simulation, the beamformer reconstruction of source $1, \hat{q}_{1}$, is given by,

$\hat{q}_{1}=q_{1}+\Delta q_{2}$.

Here, $q_{1}$ and $q_{2}$ refer to the real amplitudes of sources 1 and 2, respectively, meaning that the beamformer reconstruction of the first source $\left(\hat{q}_{1}\right)$ is given by its real value $\left(q_{1}\right)$ plus a leakage term $\left(\Delta q_{2}\right)$. The leakage magnitude, $\Delta$, thus gives a quantitative estimate of the spatial resolution; i.e. the lower the leakage, the better the spatial resolution. It is possible to show analytically (see Supplementary Material) that

$\Delta=\frac{\left\|\mathbf{l}_{2}\right\|_{F}}{\left\|\mathbf{l}_{1}\right\|_{F}}\left[\frac{R_{12}\left(1-\left(f_{1}+f_{2}\right)+f_{1} f_{2}\right)}{1-f_{1}+\left(f_{1} f_{2}-f_{2}\right) R_{12}^{2}}\right]$.

Here, $R_{12}$ is the Pearson correlation between the sensor space topographies of the two sources (i.e. the correlation between $\mathbf{l}_{1}$ and $\mathbf{l}_{2}$ ) and $f_{i}$ is a representation of signal to noise ratio, given by

$f_{i}=\frac{q_{i}^{2}\left\|\mathbf{l}_{\mathrm{i}}\right\|_{F}^{2}}{v^{2}+q_{i}^{2}\left\|\mathbf{l}_{\mathrm{i}}\right\|_{F}^{2}}$

where $v$ is the standard deviation of the sensor noise and $\|\boldsymbol{X}\|_{F}$ is the Frobenius norm of $\boldsymbol{X}$. Note that leakage depends on two factors, the correlation between the source topographies $\left(R_{12}\right)$ and SNR of the two sources. We hypothesised that OPMs would be advantageous over EEG in two ways: i) the increased spatial frequencies measurable on the scalp in OPM-MEG would reduce $R_{12}$ and ii) the increased SNR of OPMs would increase $f_{i}$. We undertook two separate simulations:

1) We measured $R_{12}$ and $\Delta$ as a function of source separation, $\delta$ (to assess spatial resolution) whilst also controlling for source depth. For this simulation we fixed source orientation to the tangential plane, and we limited $\phi$ to between $40^{\circ}$ and $50^{\circ}$. (Note that spatial resolution is maximum when sources are separated along a line perpendicular to their orientation for MEG, and parallel to their orientation in EEG. We therefore fixed $\phi$ so as not to give either EEG or MEG an advantage). Source separation and depth were allowed to vary randomly over 50,000 iterations.

2) We measured $R_{12}$ and $\Delta$ as a function of the source orientation relative to radial $(\theta)$ and the direction of separation relative to orientation (defined as the average of $\phi_{1}$ and $\phi_{2}$ ). We fixed both the source separation (to $2 \mathrm{~cm}$ ) and the source depth ( $9.6 \mathrm{~mm}$ below the brain surface). $\theta$ and $\phi_{i}$ were allowed to vary randomly over 50,000 iterations.

These two separate simulations were carried out for both EEG and OPM-MEG, allowing assessment of the fundamental limits on spatial specificity. In both simulations we assumed a random noise level of 10 $\mathrm{fT} / \sqrt{\mathrm{Hz}}$ for OPM-MEG and $120 \mathrm{nV} / \sqrt{\mathrm{Hz}}$ for EEG; we assumed a bandwidth of $100 \mathrm{~Hz}$ giving overall noise amplitudes of $100 \mathrm{fT}$ and $1.2 \mu \mathrm{V}$. These values were chosen such that the signal to noise level of EEG was similar (for a shallow radial source) to that of cryogenic MEG (for a shallow tangential source); this was based on previous findings (Goldenholz et al., 2009) (see also supplementary material). Note that a low value of $R_{12}$ and $\Delta$ indicates a significant advantage. Unlike conventional MEG, which in the case of accurate forward modelling has similar theoretical spatial resolution to EEG, we hypothesised that OPMMEG would offer fundamentally higher spatial specificity. 


\section{RESULTS}

\subsection{Motor and visual tasks with Gen-2 OPMs}

Figure 2 shows the capability of Gen-2 OPMs for measurement of beta modulation in sensorimotor cortex during the motor task. Panel (a) shows the experimental set-up, with OPMs mounted in plastic holders on a flexible (EEG-style) cap. Recall that sensor position was in accordance with a prior cryogenic-MEG recording, the result from which is also shown inset. Panels (b)(i)-(iii) show trial-averaged TFSs, acquired with the OPMs' outer casing at stand-off distances of 0,8 and $18 \mathrm{~mm}$ from the scalp surface, respectively. Panel (iv) shows phantom data for comparison. The advantages of placing OPMs closer to the scalp can be seen clearly, with the magnitude of the signals of interest decreasing non-linearly with increasing distance, as expected. Note that the 18-mm stand-off is approximately where a cryogenic pick-up coil might be placed. Panel (c) shows an example of single-trial beta-band responses; the trace shows a representative $100 \mathrm{~s}$ segment of data, filtered $13-20 \mathrm{~Hz}$, and the green shading shows a block of 10 trials in which the subject made 10 finger abductions. The pink shading shows a rest-block in which the participant made no hand movements. Trial onsets are marked with a black circle. Notice that single-trial events can be seen clearly. Panel (d) quantifies SNR. Note that SNR was measured independently in three response windows: the movement-related beta decrease ( $0.1 \mathrm{~s}$ to $1.0 \mathrm{~s}$ relative to trial onset), the beta rebound ( $1.5 \mathrm{~s}$ to $2.4 \mathrm{~s}$ ), and the resting phase of the experiment ( $2.1 \mathrm{~s}$ to $3.0 \mathrm{~s}$ in the rest trials). As expected, the SNR decreases with stand-off distance for real brain signals (rebound and rest): SNR values (mean \pm standard deviation) range from $50.5 \pm 36.6,18.2 \pm 13.2$ to $6.5 \pm 4.3$ in the rebound window, and from $11.5 \pm 12.9,4.5 \pm 2.9$ to $1.9 \pm 1.3$ in the rest blocks, at $0 \mathrm{~mm}, 8 \mathrm{~mm}$ and $18 \mathrm{~mm}$, respectively. In contrast, SNR shows little change with no measurable brain response (during finger movement): at $0 \mathrm{~mm}, 8 \mathrm{~mm}$ and $18 \mathrm{~mm}$ from the scalp, SNR values are 2.5 \pm 2.0 , $3.1 \pm 1.7$ to $1.7 \pm 1.2$, respectively. This is a potentially useful means to differentiate real brain activity from noise, and this idea will be revisited in the Discussion. Equivalent results for each of the magnetometers are shown in Figure S1 in the Supplementary Material.

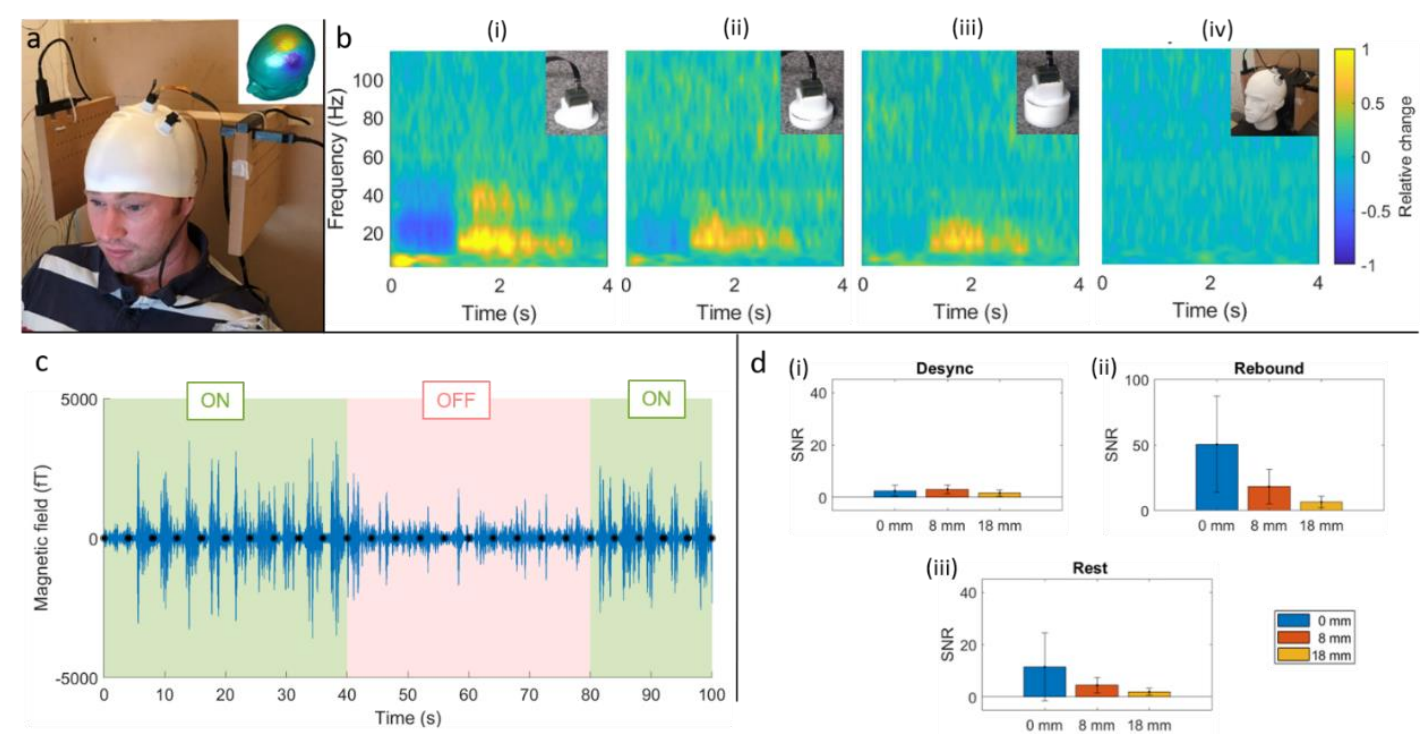

Figure 2: Beta-band responses in primary motor cortex. a) Experimental set-up; the Gen-2 OPMs were positioned on the scalp based on a field map from a previous cryogenic MEG experiment, which is shown inset. The 4 Gen-1 OPMs used in the reference array can be seen around the subject. b) TFSs showing oscillatory modulation with the sensors mounted at distances of $0 \mathrm{~mm}$ (i), 8 $\mathrm{mm}$ (ii) and $18 \mathrm{~mm}$ (iii) from the scalp surface. (iv) shows a phantom measurement for comparison. All results are extracted from a planar gradiometer formulation of the two sensors. c) Representative single-trial beta responses during task (green) and rest (pink) blocks. Note that the task blocks contains ten trials of finger abduction (marked with black circles). d) Quantification of the (singletrial) SNR during the movement-induced beta-band decrease (i), the beta rebound (ii) and at rest, i.e. resting beta-band level (iii). For magnetometer data, see Supplementary Material. 
Figure 3 is equivalent to Figure 2 but shows results of the visual paradigm. The experimental set-up is shown in panel (a) and the TFSs are shown in panel (b). Panel (c), shows single-trial responses, with the green-shaded regions representing periods when the visual stimulus was on the screen. In panel (d), SNR was measured in the $0.2 \mathrm{~s}$ to $2.2 \mathrm{~s}$ time window (relative to onset of visual stimulation) and values range from $3.8 \pm 1.0,2.2 \pm 0.5$ to $1.6 \pm 0.2$ at 0,8 and $18 \mathrm{~mm}$ from the scalp, respectively. It is noteworthy that the response of interest here is at a higher frequency, and lower amplitude, than the beta modulation shown in Figure 1 ( $60-80 \mathrm{~Hz})$. Nevertheless, the OPMs were able to capture it, even in single trials. Note also the expected alpha band response in the TFSs.

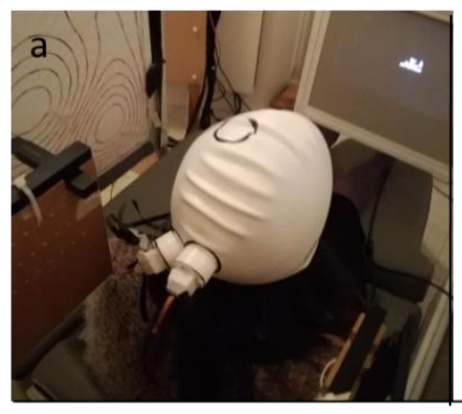

b

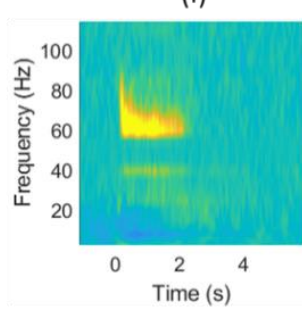

(ii)

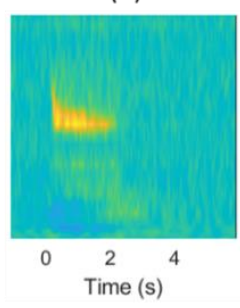

(iii)

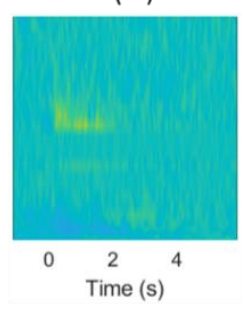

(iv)

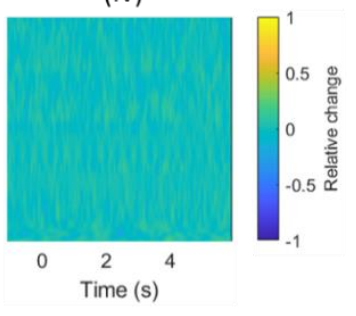

C

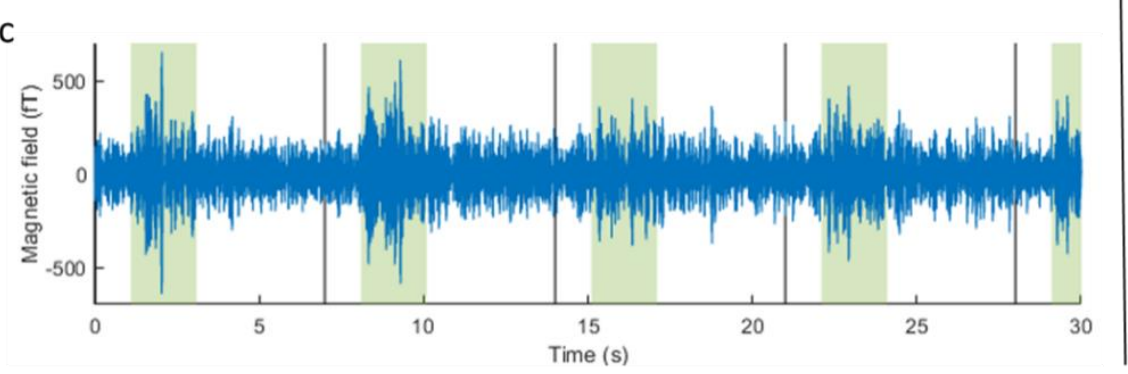

d

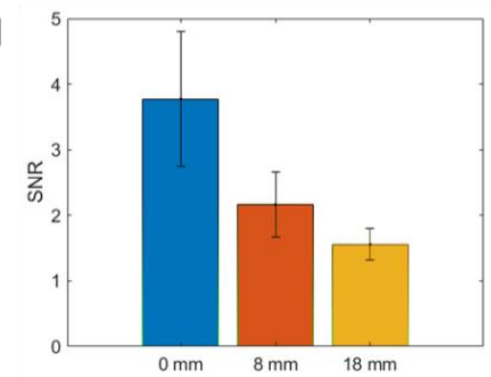

Figure 3: Gamma-band responses in primary visual cortex. a) Experimental set-up. b) TFSs showing gamma modulation with the sensor mounted at distances of $0 \mathrm{~mm}$ (i), $8 \mathrm{~mm}$ (ii), $18 \mathrm{~mm}$ (iii) from the head. Note also a stimulus induced drop in alpha (8-13 $\mathrm{Hz}$ ) band amplitude during stimulation is apparent. (iv) shows a phantom recording for comparison. c) Representative single-trial gamma responses with periods of visual stimulation (stimulation windows highlighted in shaded green). d) Quantification of the (single-trial) SNR during stimulation for each sensor distance.

\subsection{Concurrent EEG/MEG - Stationary}

Figure 4a shows the experimental set-up for concurrent EEG/MEG. The EEG cap was fitted to the participant's head and overlaid with a second fabric cap, upon which holders for the OPMs were mounted. The inset plot shows a colour map depicting the beta-band response at each EEG electrode, highlighting with a red circle the location of the EEG channel that gave the highest signal (C3). Figure $4 b$ shows TFSs from MEG (left) and EEG (right) responses to finger abduction. The similarity between the two responses is clear. Also clear is the increased SNR of the OPM-MEG measurement, which here manifests as a lower fractional change in signal, relative to baseline in the EEG channel with the largest response. Figure 4c shows trial-averaged line graphs of the beta modulation; the blue line shows the average of the task trials and the red line shows an average over rest trials (where no response is expected). Again, a clear response can be seen which is similar in both modalities. However, the increased fractional beta change in the OPM-MEG data is apparent: this was $2.2 \pm 1.2$ for the OPM gradiometer $(0.7 \pm 0.4,1.2 \pm 0.7$ for each of the magnetometers) and $1.0 \pm 0.3$ for the best EEG electrode. Finally, Figure $4 \mathrm{~d}$ shows single-trial responses from both modalities in the $13-20 \mathrm{~Hz}$ band. 

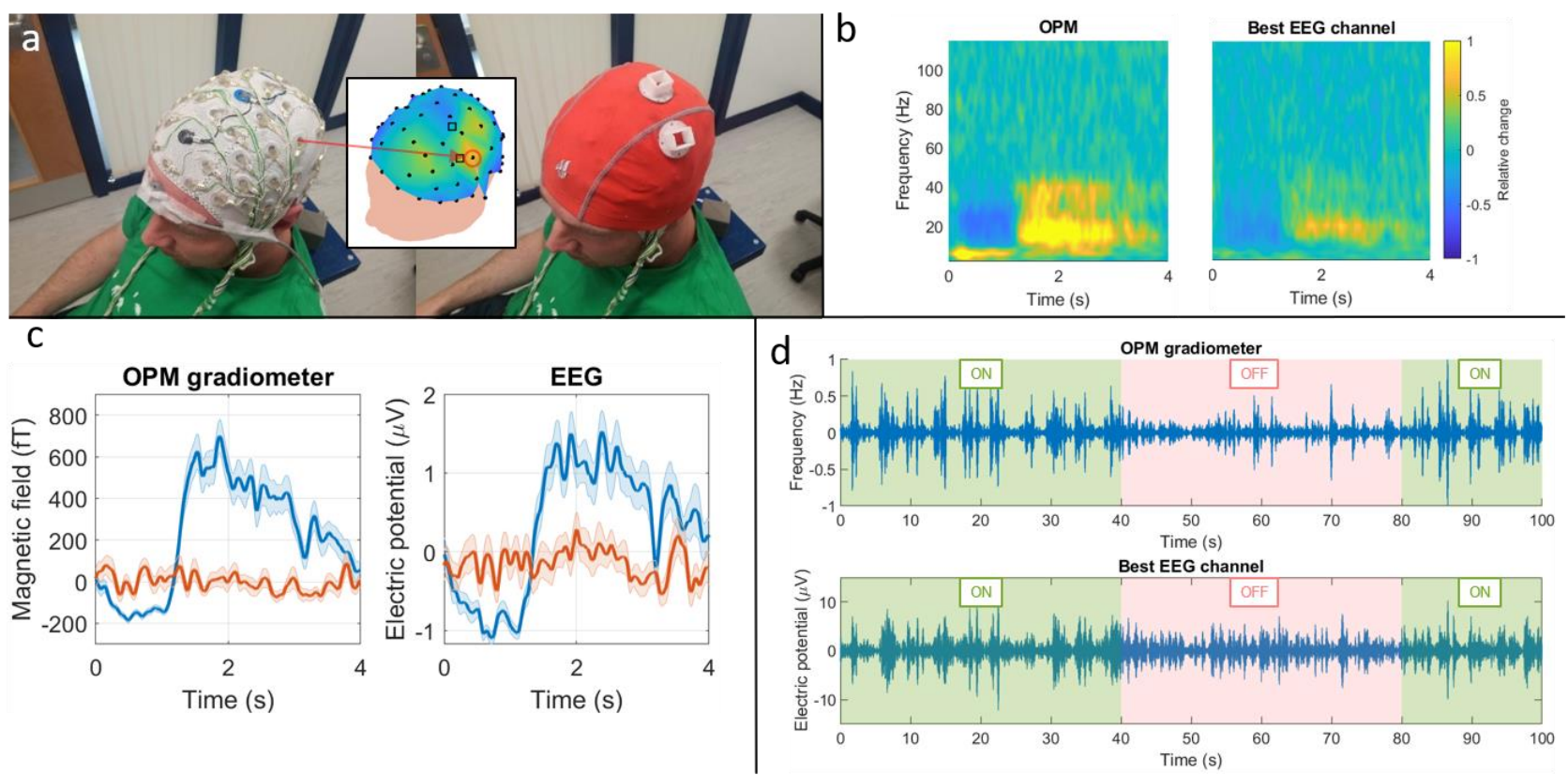

Figure 4: Simultaneous EEG/MEG - Task. a) Experimental set-up showing photographs of the EEG cap, mounted on the subject's head and overlaid with a second flexible cap housing the OPMs. The central plot shows the EEG (black dots) and OPM channels (black squares) on the subject's head, and the EEG channel (C3) that showed the highest beta-band response is circled in red. $b$ ) TFSs showing responses in OPM-MEG and EEG. c) Line plots showing the baseline-corrected beta-band oscillatory amplitude, averaged across task trials (blue) and rest trials (red). d) Single-trial responses in the 13-20 Hz band, for OPM (top) and EEG (bottom).

Results of the resting state experiments are shown in Figure 5. Panels (a) and (b) show the PSD of the EEG signal (from electrode C3) and MEG signal (from OPM gradiometer), respectively. All line plots correspond to the average over the five four-minute blocks and shaded areas represent standard deviation across runs. In panel (a), the blue line shows the PSD of resting state data collected when subject was only wearing the EEG cap. In purple, the equivalent PSD, acquired in the presence of the OPMs is shown. Likewise, in panel (b), the blue line shows OPM data without EEG, and the purple line shows the case with EEG. Importantly, no marked differences can be seen between the spectra, meaning each modality is unaffected by the presence of the other. The slight increase in oscillatory power at high frequency for the OPM case (in the presence of EEG) is caused by one of the five resting state runs, which increased the average value. 

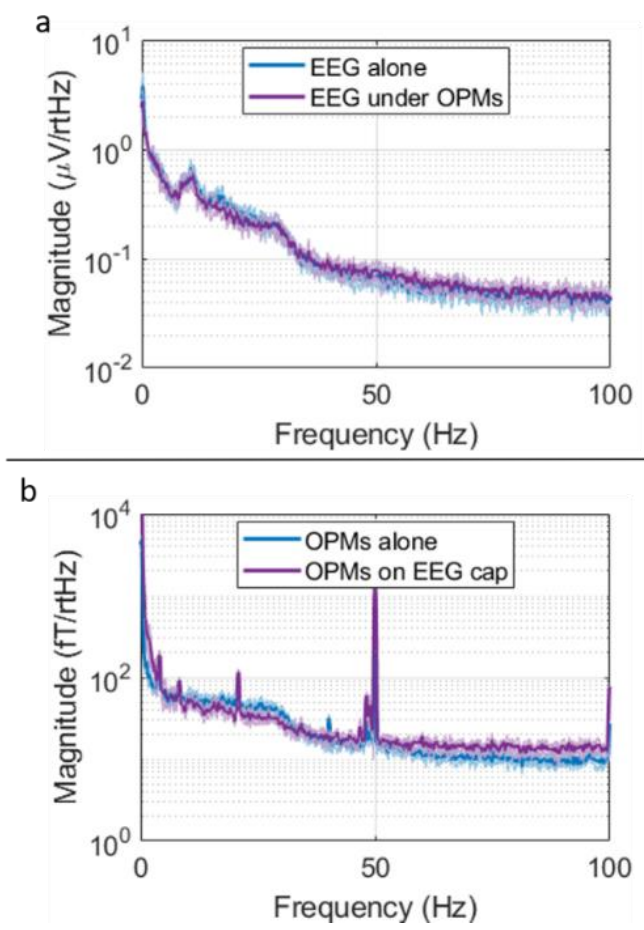

Figure 5: Simultaneous EEG/MEG - Resting state. a) Power spectral density of resting state EEG signal when collected alone (blue) and concurrently with the OPMs (purple). b) Equivalent PSD for the OPM planar gradiometer when collected without (blue) and with (purple) the EEG cap. (For magnetometer results see Supplementary Material).

\subsection{Concurrent EEG/MEG - Moving}

Figure 6 shows the effect of subject head movement on EEG and MEG measurements. Figure 6a shows TFSs for OPM-MEG (left) and EEG (right) in the case where the subject made small head movements. Figure $6 \mathrm{~b}$ shows the equivalent metrics in the presence of large head movements. Notice that the OPM-MEG response is largely unchanged by the addition of subject movement, but the EEG response is contaminated with artefacts in a frequency range stretching from the beta band to beyond $100 \mathrm{~Hz}$. This frequency range is characteristic of the electromyogram. Figure $6 \mathrm{~d}$ shows example gamma-band envelopes, for a single OPM sensor (i.e. not the synthesised gradiometer) (upper panel) and a single EEG electrode (lower panel). Here, raw data have been filtered to the $75-100 \mathrm{~Hz}$ band, the envelope derived, and plotted over the entire time course of the experiment. The blue line shows the case where the subject remained still, while the yellow line shows the case where the subject was moving. Note that the envelope of the OPM is largely flat in both cases, but head movement degrades the quality of the EEG measurement. This effect is quantified in the bar chart in Figure $6 c$, which shows our interference metric for OPM-MEG and EEG. Note that we include both a single OPM magnetometer and our synthesised gradiometer (OPG) for comparison. All three experimental cases - no movement, small movements and large movements - are shown. The error bars represent standard deviation across trials. Clearly, even in the case of small movement, EEG data begin to degrade and this becomes worse in the case of large movement. However, both the OPM magnetometer and gradiometer metrics remain relatively unaffected by the movement. These data show that EEG and MEG measurements differ in their sensitivity to head movement. 


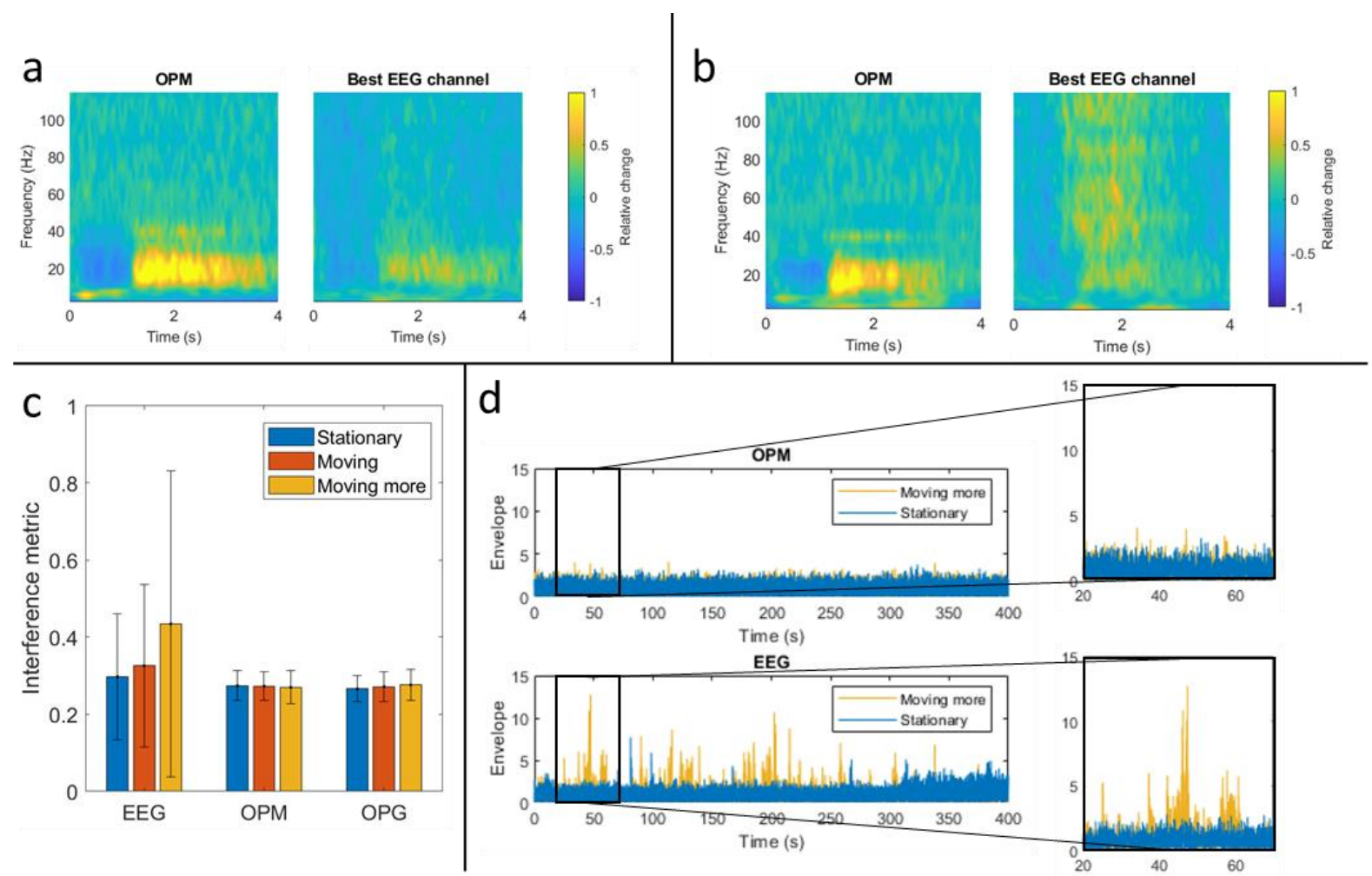

Figure 6: The effects of movement during task. a) TFSs from OPM-MEG and EEG in the case where the subject was asked to make small, natural head movements. b) TFSs from OPM-MEG and EEG in the presence of large head movements. Notice that artefacts stretching from the high beta to the gamma band are apparent, and these responses are characteristic of electromyogram data. $c$ ) Quantification of the interference metric for EEG and OPM-MEG (for both magnetometer and gradiometer (OPG)). Error bars show standard deviation across trials. d) High-frequency envelopes recorded from a single OPM (top), and EEG electrode (bottom). Blue shows the case when a subject was asked to keep still and yellow shows the case for large head movements.

In Figure 7, the results of resting state experiments in the presence of movement are shown. Panel (a) shows averaged PSD (across runs) for EEG (top) and MEG (bottom). In both cases, blue represents the static case and red is the average of the moving runs. Zoomed-in plots show PSD between 10-35 Hz. An increase (from approximately $20 \mathrm{~Hz}$ ) can be seen clearly in the EEG spectrum in the presence of movement, while no such difference is apparent in the MEG spectra. This is in agreement with results in Figure 6 showing that EEG data, upwards in frequency from the beta band, are adversely affected by movement. Interestingly, the MEG spectra shows a reduced power around the beta-band range during the moving runs, perhaps implying the head movement is causing a drop in beta-band signal (desynchronization). It also can be noticed the higher power at low frequencies $(<5 \mathrm{~Hz})$ for the MEG signal during the moving runs, compared to the static runs. This might be due to small vibration of the sensors caused by the movement. Panel (b) shows maps of beta-band envelope correlation between all EEG channels and the OPM gradiometer during static runs (top) and moving runs (bottom). During stationary runs, there is an expected high correlation between the OPM gradiometer and EEG electrodes located around the left central/parietal region (with effects stretching to the right parietal areas likely reflective of genuine functional connectivity between sensorimotor areas). However, this correlation decreases when data from the moving runs are used, suggesting that beta-band data are compromised in the presence of natural head movements. 
a
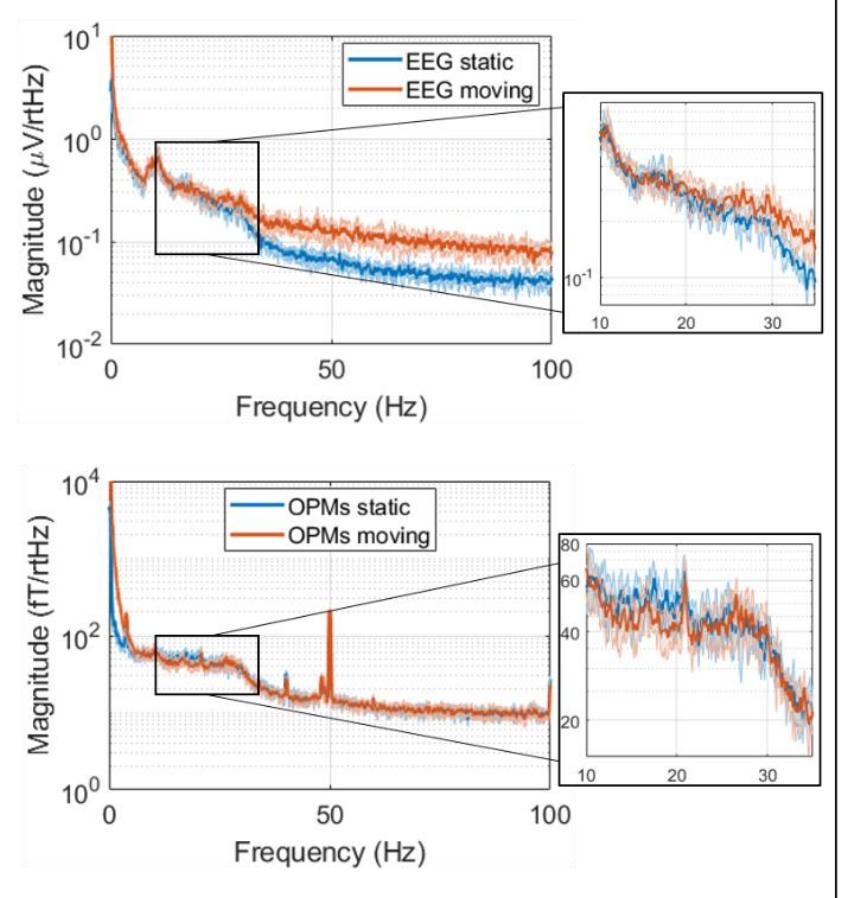

b
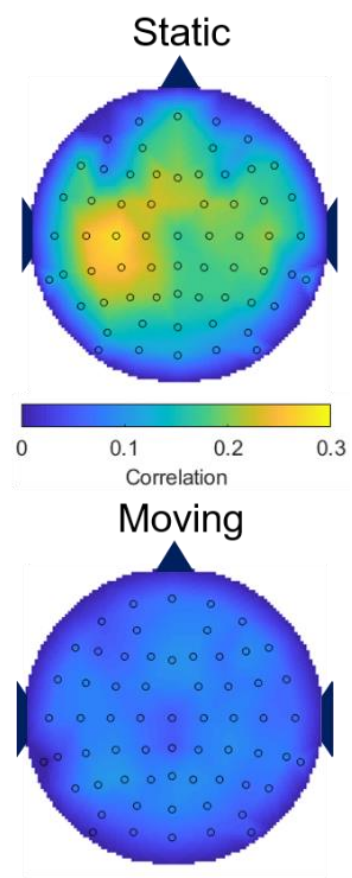

Figure 7: The effects of movement during resting state. a) Power spectral density plots for EEG (top) and OPM planar gradiometer (bottom) when collected while the subject was stationary (blue) and moving (red). b) Beta-band envelope correlation between all the EEG channels and the OPM planar gradiometer in the stationary (top plot) and moving (bottom plot) conditions.

\subsection{Spatial resolution simulations}

Figure 8 shows the results of the simulations on spatial specificity. The simulation set-up is shown in Figure 8a, whilst Figure $8 \mathrm{~b}$ shows example EEG (left) and MEG (right) field patterns on a spherical surface. The source locations are shown by the black lines and source orientations are shown by the red lines. Notice that the EEG field pattern is considerably more diffuse (i.e. contains lower spatial frequencies) at the surface upon which it was measured (the scalp) compared to the OPM-MEG spatial topography (measured $6 \mathrm{~mm}$ above the scalp). Figure $8 c$ shows the consequence of these increased spatial frequencies: in (i) and (ii) the correlation between source topographies $\left(R_{12}\right)$ is plotted as a function of both source separation and source depth, for EEG and MEG, respectively. It is clear that the forward fields are less correlated for OPM-MEG than for EEG for an equivalent source separation; whilst this is true for all sources, the advantage does lessen with increasing source depth. In (iii) and (iv) $R_{12}$ is plotted against source orientation and the direction of source separation, for EEG and OPM-MEG, respectively. Here again we see the distinct advantage of OPM-MEG with generally lower forward field correlation. However, as would be expected, EEG shows advantages when the direction of source separation is parallel to the source orientation $(\phi=0$ or $\phi=180)$.

Measurement of field correlation $\left(R_{12}\right)$ is solely a property of the spatial topographies of EEG and MEG forward fields and fails to account for SNR of the measurements. For this reason, in Figure $6 \mathrm{~d}$ we plot the estimated spatial leakage, $\Delta$, as a function of source separation and depth ((i) and (ii), for EEG and MEG respectively). Spatial leakage is plotted as a function of source orientation and direction of separation, in (iii) and (iv), for EEG and MEG respectively. Here, the improved SNR of OPM-MEG is accounted for and as a result, for tangential sources the advantages of OPM-MEG over EEG in terms of spatial resolution become even more striking (with the leakage term exhibiting a markedly lower magnitude for OPMs). However, the complementary nature of MEG and EEG remains apparent, specifically with EEG still offering significant advantages for sources close to radial. (See also supplementary material). 

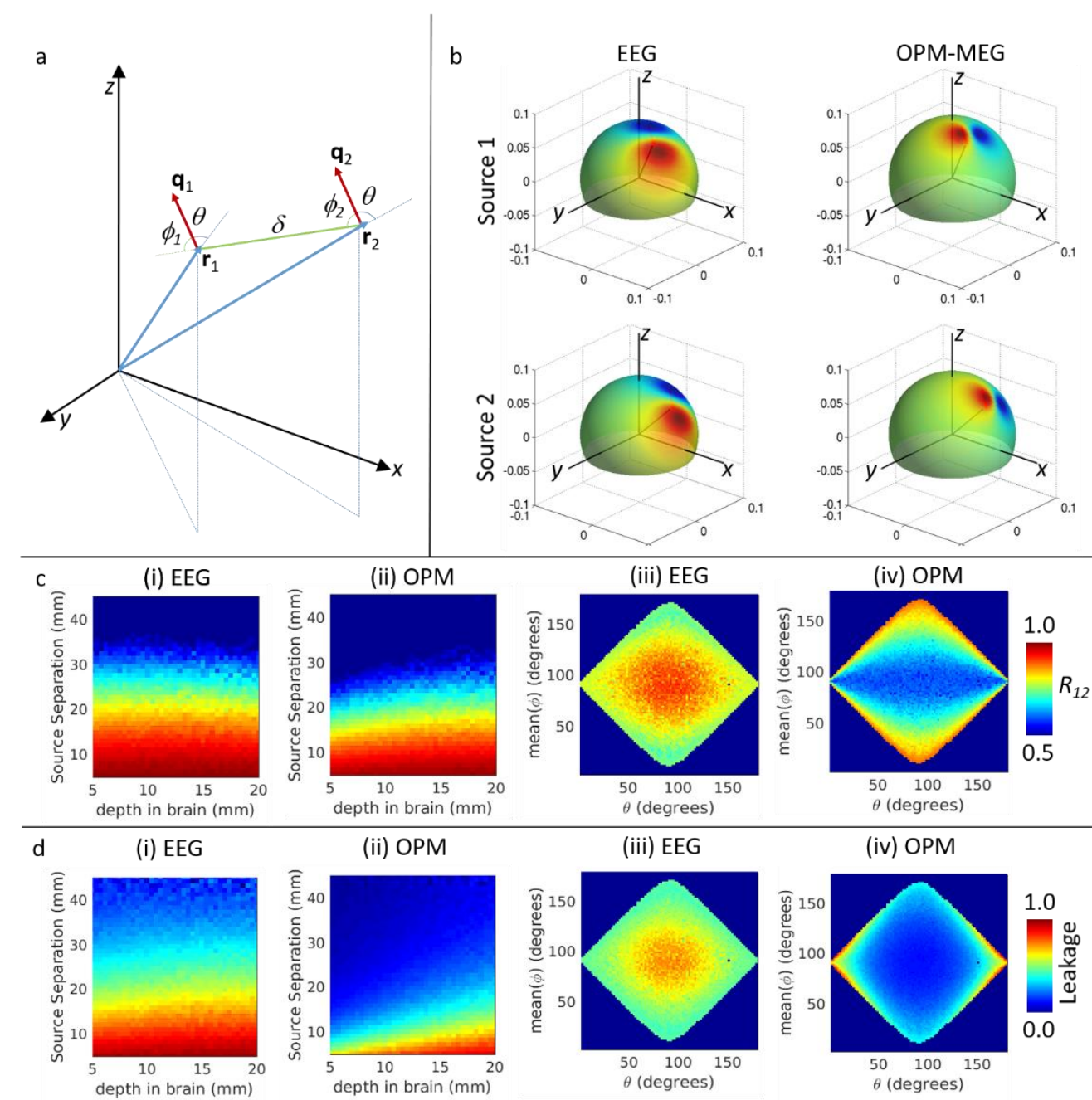

Figure 8: Spatial resolution simulations. a) Schematic diagram showing the two simulated sources. b) Example forward fields simulated for EEG (left) and OPM-MEG (right). Blue lines show source positions ( $\boldsymbol{r}_{1}$ and $\boldsymbol{r}_{2}$ in (a)); red lines show source orientations $\left(\boldsymbol{q}_{1}\right.$ and $\boldsymbol{q}_{2}$ in (a)). b) Upper plots show source 1, lower plots show source 2. Notice that in EEG field patterns are made diffuse by the low conductivity of the skull. However, field patterns measurable using OPM-MEG contain higher spatial frequencies. c) Parametric variation of spatial correlation of forward fields for sources 1 and 2 (i.e. $R_{12}$ ). Left two panels show variation with source depth separation, for EEG (i) and OPM-MEG (ii). Right 2 panels show variation with $\theta$ (mean angle to radial) and $\phi$. d) Parametric variation of estimated leakage (i.e. $\Delta$ ) between sources 1 and 2. Panels as in (c). (See also supplementary material for results on conventional MEG.)

\section{DISCUSSION}

Recent studies (Boto et al., 2018; Holmes et al., 2018; Tierney et al., 2018) have shown the significant potential of OPMs for measuring MEG signals using wearable instrumentation, in which subjects are considerably less constrained during data acquisition. However, to date, commercially-available sensors have remained relatively large $\left(1.3 \times 1.9 \times 11.0 \mathrm{~cm}^{3}\right)$, and the weight of cabling has meant that scaling to a system with a high channel count would likely be impractical due to the torque generated on the participants' head by the weight of the system wiring. This would be particularly problematic in paediatric studies. However, recent years have seen vast progress in miniaturisation of OPM sensors (Borna et al., 2017; Jiménez-Martínez and Knappe, 2017; Kamada et al., 2015; Osborne et al., 2018; Sheng et al., 2017) and this work has ultimately 
led to the recent (2018) commercialisation of a new generation of smaller and lighter OPMs. This paper, to our knowledge, is the first to demonstrate the use of these Gen-2 commercial OPMs (QuSpin Inc., CO, USA) in a wearable system. These new sensors are smaller and lighter than Gen-1 OPMs, and with lighter cabling, enabling easier mounting on the head and better facilitating natural movement. We were able to mount the sensors on a flexible (EEG-like) cap, fabricating a prototype system that could readily be scaled to a high sensor count with whole-head coverage, without the problems of cable weight or sensor size. This represents a significant step forward in the practicality of wearable MEG as a neuroimaging tool. Indeed, in a fully fabricated (e.g. 64-channel) system, the effect of this switch from Gen-1 to Gen-2 sensors would be that system weight decreases from $2 \mathrm{~kg}$ to $\sim 300 \mathrm{~g}$, and cable weight from $33 \mathrm{~g} / \mathrm{m}$ to $3.3 \mathrm{~g} / \mathrm{m}$. Our results show the suitability of this new generation of sensors for MEG measurements, generating high quality data from primary visual and motor cortices. We showed that the close proximity to the brain, coupled with the high sensitivity of the sensors, enabled high fidelity measurements even when looking at individual trials. This in itself potentially opens up new avenues of research in e.g. motor learning, wherein the morphology of individual responses is of interest.

In Figures 2 and 3, we experimentally demonstrated the expected non-linear fall-off of signal magnitude with distance from the scalp. This not only serves as a reminder of the advantages of 'on-scalp' recording, but also points the way towards potentially improved array design. As sensors move away from the head, brain signals are reduced in magnitude, whilst signals of no interest - interference - are likely to either remain constant or increase. This offers a means to differentiate real signals from interference; indeed this is the basis of hard-wired axial gradiometer designs used in conventional (SQUID-based) MEG systems and it is tempting to suggest that OPM gradiometers could be designed along similar lines (Sheng et al., 2017), would be advantageous. However, the majority of OPMs are inherently magnetometers. Because of their small size, it is possible to envisage the positioning of two units, one on the scalp surface, and one a short (e.g. 3-5 cm) distance away, and digital subtraction of the two signals could generate an axial gradiometer signal which would cancel interference. However, the subtraction would also increase random noise (by $\sqrt{ } 2$ ), and this is undesirable. Alternatively, an array could be designed in which these small sensors are operated in a gradiometer arrangement, but independently measuring two signals and using source localisation algorithms (e.g. beamforming) to exploit the fact that signals of interest decay with distance. This would potentially provide greater information content, a powerful way to reject interference (compared to a hardwired gradiometer), and perhaps better spatial resolution. This type of array design, which can only be realised using small OPMs, should be the subject of future work.

The introduction of small packaged MEG sensors enabled conception of a wearable imaging system comprising both MEG and EEG measurement. MEG and EEG are complementary for two reasons: first, MEG (conventional or OPM) is largely insensitive to sources oriented radial to the scalp surface, whereas EEG has its best sensitivity for these same radial sources. This means that for sources with small tangential components, EEG offers better SNR. Secondly, for tangential dipoles, both MEG and EEG have greatest spatial resolution when the sources are separated along a direction joining the two lobes of the measured dipolar field pattern. Because the field patterns for MEG and EEG are orthogonal, if source separation is perpendicular to the source orientation, MEG offers a better spatial differentiation, whereas if it is parallel, EEG offers a better differentiation. These simple concepts make a hybrid device theoretically attractive. Here, our experimental results (Figures 4 and 5) showed that unmodified commercial OPMs can be operated in close proximity to commercial $\mathrm{Ag} / \mathrm{AgCl} \mathrm{EEG}$ electrodes, with no appreciable loss in data quality in either modality. This paves the way for the design of a multi-channel OPM-MEG-EEG system, with scalp-mounted sensors, on a flexible cap. Indeed, an EEG electrode integrated into an OPM - i.e. a hybrid sensor unit potentially offers an attractive solution to concurrent recordings. 
Although concurrent EEG/MEG is clearly an attractive proposition, our results also show significant advantages of OPM-MEG measures when compared to EEG. The most often quoted advantage of MEG is improved spatial resolution, however this is not an inherent property of the technique, but rather a data modelling issue brought about because the EEG forward problem is difficult to solve. Here, our simulations ignored these modelling difficulties and assumed that one could solve both the EEG and MEG forward solutions perfectly, offering a window on the theoretical limits of spatial resolution. We showed (see also Supplementary Figures) that EEG and conventional MEG have similar limits, however OPM-MEG has significant advantages: these result from the higher spatial frequencies available in OPM-MEG scalp topographies. In EEG, field patterns are made spatially diffuse by a spreading of electrical potentials caused by the low conductivity of the skull. In conventional MEG, a similar diffusivity is generated as a result of the sensors being cited a long way from the head. Consequently, the two measured field patterns have similar spatial properties and to our ability to separate two sources is therefore similar (modelling issues notwithstanding). However, in OPM-MEG, the sensors are closer to the brain, and the skull has little effect, meaning that higher spatial frequencies can be captured at the scalp surface which help to differentiate nearby sources. Coupled with this, the SNR of OPM-MEG is higher than that of EEG. These two effects combine to produce a result that the theoretical limit on spatial resolution (for a beamformer) is higher for OPM-MEG than either EEG or conventional-MEG; this is no longer a modelling issue, but rather a fundamental property of the measurement (i.e. even if the MEG and EEG forward models were perfectly known, OPMMEG will always have better spatial resolution). This is the case for almost all sources in the brain. However, as would be expected EEG still offers advantages when sources are close to radial in orientation.

A significant limitation of EEG is sensitivity to artefacts generated by electrical activity in muscles. This is thought to be a result of volume conduction through tissue, and has been shown in previous papers. For example, Whitham et al., (2007) recorded EEG data with and without neuromuscular blockade and showed that artefacts at frequencies above $\sim 20 \mathrm{~Hz}$ are generated by muscle activity. Other literature (Boto et al., 2018; Claus et al., 2012; Muthukumaraswamy, 2013; Zimmermann and Scharein, 2004) has suggested that because the magnetic field generated by muscle falls off rapidly with distance, MEG is less susceptible to such effects. Here, our wearable EEG/MEG system offered an opportunity for the first direct comparison of EEG and MEG signals in a subject who was asked to make natural head movements. Our results showed that with the head stationary, EEG and OPM-MEG both offered high-fidelity electrophysiological measurement at the channel level (albeit with OPMs demonstrating higher SNR). However, even in the presence of small movement, the EEG signal, particularly in the gamma band, degrades significantly and in the presence of large movement, the muscle artefact obfuscated signals in the beta band and above. Conversely, the OPM-MEG signal remained measurable with little degradation of the SNR. Indeed this was shown clearly in Figures 6 and 7 with an approximately 9-fold variability in gamma-band measurements in EEG compared to MEG.

Bringing together the above arguments, combining EEG and OPM-MEG offers a wearable neuroimaging system which, ostensibly, allows one to exploit the complementarity of the two modalities. However, from a spatial specificity point of view, our simulations show that the addition of EEG will only help when sources are very close to being radial; otherwise OPM-MEG offers fundamentally improved spatial resolution. More importantly, the major advantage of wearable neuroimaging is that subjects can move during scanning. However, our results show that when subject movement is allowed (or indeed encouraged), muscle artefacts are clearly a major problem for EEG - degrading the quality of data in the $>20 \mathrm{~Hz}$ frequency band. It follows that, in practice, the benefits of concurrent recording over OPM-MEG alone, particularly given the relatively high set-up time for EEG, are questionable. With this in mind, perhaps the best argument for concurrent measures are from a clinical perspective: patient evaluation (in e.g. epilepsy and sleep scoring) 
using EEG has been undertaken for many years, and concurrent OPM-MEG/EEG measurements potentially offer an approach in which clinicians are offered the significant advantages of OPM-MEG, without the need to give up 'tried and tested' EEG metrics. Of course EEG alone remains somewhat simpler; EEG measures can be carried inside a simple faraday cage, or even in the absence of shielding whereas MEG (OPMs or otherwise) requires a MSR which, at present, comprise multiple layers of mu-metal which can be heavy and costly. However, the higher signal afforded by OPMs, coupled with the introduction of active shielding systems (e.g. Holmes et al., (2018); livanainen et al., (2019)), could bring down the size and cost of MEG shielding in the future.

We acknowledge that a perceived limitation of the present paper is potentially that we have acquired data in a small number of subjects. Indeed, we have opted to acquire multiple 'test-retest' datasets in single individuals rather than a large multi-subject dataset. We chose this approach for two reasons: first, the effects sought, both neurological (e.g. beta and gamma modulation) and artefactual (the electromyogram) are extremely well characterised - they can be measured in individual subjects and are well known to generalise across a cohort. Second, we reasoned that test-retest in a single subject would minimise variability across datasets, and better show the effects of e.g. muscle artefacts on brain signals. This said, running OPMMEG across large cohorts remains something that has not yet been published, and should be the topic of future work. We also acknowledge that the ultimate success of the system designed here depends critically on the scalability of OPM units, and our ability to use a dense array to cover the whole scalp and capture the high spatial harmonics of the field patterns shown in our simulations. Scalability is currently a work in progress, but at the time of writing OPMs can already be generated efficiently in reasonable numbers. The future use of microelectromechanical systems (MEMS) technology for cell fabrication, and potentially advanced multi-material additive manufacturing, might increase the speed of production and lower costs. Operation of a whole-head array, with sensors in sufficiently close proximity to capture the highest spatial frequencies of the measurable fields, presents its own challenges. Specifically, the on-board sensor coils generate both static and time-varying fields inside the cell that are used to cancel background field and provide directional sensitivity, respectively. At present, these fields stray outside the OPM housing and can interfere with measurements within other sensor units in close proximity. Although understanding and eliminating this crosstalk is critical, the problem is readily addressed either by advanced forward models, or potentially new on-board coil designs that generate known fields in the cell, and zero field outside. The scalability of the system shown here is therefore a significant, but solvable, challenge and there are, in principle, no fundamental barriers to the introduction of high sensor density wearable OPM-MEG systems for use in neuroscientific research or clinical evaluation.

Finally, one question is how the new OPMs used here compare to SQUID-based sensors used in conventional MEG. Experimental comparisons between SQUIDs and OPMs have been made previously, with a general finding that whilst the OPM noise level is higher, by virtue of closer positioning to the brain, the OPM signal is also higher. This resulted in, for example, Boto et al., (2017) showing an improvement in SNR of a factor of 1.99 for OPMs compared to SQUIDs; livanainen et al., (2019b) make a similar comparison using measured visually-induced gamma oscillations. A complete source-space comparison of OPM-MEG using Gen-2 sensors, and conventional MEG remains an important question, and should be the topic of future work, in particular when higher sensor counts are available.

\section{CONCLUSION}

OPMs are nascent technology for measurement of the small biomagnetic fields generated by the body. Here we have demonstrated that a new generation of smaller and lighter OPMs can be mounted in an EEG-like cap, offering a simple and attractive solution to measuring the MEG signal using a wearable system. We have 
shown that these microfabricated sensors can be used in conjunction with traditional EEG, offering the potential for a hybrid system that could exploit the complementarity of the two modalities. However, we also showed that OPM-MEG has significant advantages over EEG, being less sensitive to artefacts generated by muscles during natural head movement and offering fundamentally better spatial specificity for almost all sources in the brain. OPM technology thus has significant potential to supplement, or potentially replace, EEG as the best option for wearable functional neuroimaging.

\section{ACKNOWLEDGEMENTS}

We would like to thank Dr Lauri Parkkonen for providing the visual stimulus used to generate the data in Figure 3. This work was supported by a Wellcome Collaborative Award in Science (203257/Z/16/Z and 203257/B/16/Z) awarded to GRB, RB and MJB. It was also supported by the UK Quantum Technology Hub for Sensors and Metrology, funded by the Engineering and Physical Sciences Research Council (EPSRC) (EP/M013294/1). Funding from EPSRC and Medical Research Council (MRC) (grant number EP/L016052/1) provided a Ph.D. studentship for GR through the Oxford Nottingham Biomedical Imaging EPSRC and MRC Centre for Doctoral Training.

\section{CONFLICT OF INTEREST}

Co-author Vishal Shah is founding director of QuSpin - the commercial entity selling OPM magnetometers. QuSpin built the sensors used here and advised on the system design and operation, but played no part in the subsequent measurements or data analysis. This work was funded by a Wellcome award which involves a collaboration agreement with QuSpin.

\section{REFERENCES}

Allred, J.C., Lyman, R.N., Kornack, T.W., Romalis, M. V., 2002. High-Sensitivity Atomic Magnetometer Unaffected by Spin-Exchange Relaxation. Phys. Rev. Lett. 89, 130801. https://doi.org/10.1103/PhysRevLett.89.130801

Babiloni, F., Babiloni, C., Carducci, F., Romani, G.L., Rossini, P.M., Angelone, L.M., Cincotti, F., 2004. Multimodal Integration of EEG and MEG Data: A Simulation Study with Variable Signal-to-Noise Ratio and Number of Sensors. Hum. Brain Mapp. https://doi.org/10.1002/hbm.20011

Babiloni, F., Carducci, F., Cincotti, F., Gratta, C. Del, Pizzella, V., Romani, G.L., Rossini, P.M., Tecchio, F., Babiloni, C., 2001. Linear inverse source estimate of combined EEG and MEG data related to voluntary movements. Hum. Brain Mapp. https://doi.org/10.1002/hbm.1052

Baillet, S., 2017. Magnetoencephalography for brain electrophysiology and imaging. Nat. Neurosci. 20, 327339. https://doi.org/10.1038/nn.4504

Baillet, S., Garnero, L., Marin, G., Hugonin, J.P., 1999. Combined MEG and EEG source imaging by minimization of mutual information. IEEE Trans. Biomed. Eng. 46, 522-534. https://doi.org/10.1109/10.759053

Berger, H., 1929. Über das Elektrenkephalogramm des Menschen. Arch. Psychiatr. Nervenkr. 87, 527-570.

Borna, A., Carter, T.R., Goldberg, J.D., Colombo, A.P., Jau, Y.Y., Berry, C., McKay, J., Stephen, J., Weisend, M., Schwindt, P.D.D., 2017. A 20-channel magnetoencephalography system based on optically pumped magnetometers. Phys. Med. Biol. 62, 8909-8923. https://doi.org/10.1088/1361- 
Boto, E., Bowtell, R., Krüger, P., Fromhold, T.M., Morris, P.G., Meyer, S.S., Barnes, G.R., Brookes, M.J., 2016. On the potential of a new generation of magnetometers for MEG: A beamformer simulation study. PLoS One 11, e0157655. https://doi.org/10.1371/journal.pone.0157655

Boto, E., Holmes, N., Leggett, J., Roberts, G., Shah, V., Meyer, S.S., Muñoz, L.D., Mullinger, K.J., Tierney, T.M., Bestmann, S., Barnes, G.R., Bowtell, R., Brookes, M.J., 2018. Moving magnetoencephalography towards real-world applications with a wearable system. Nature 555, 657-661. https://doi.org/10.1038/nature26147

Boto, E., Meyer, S.S., Shah, V., Alem, O., Knappe, S., Kruger, P., Fromhold, T.M., Lim, M., Glover, P.M., Morris, P.G., Bowtell, R., Barnes, G.R., Brookes, M.J., 2017. A new generation of magnetoencephalography: Room temperature measurements using optically-pumped magnetometers. Neuroimage 149, 404-414. https://doi.org/10.1016/j.neuroimage.2017.01.034

Claus, S., Velis, D., Lopes da Silva, F.H., Viergever, M.A., Kalitzin, S., 2012. High frequency spectral components after Secobarbital: The contribution of muscular origin-A study with MEG/EEG. Epilepsy Res. 100, 132-141. https://doi.org/10.1016/j.eplepsyres.2012.02.002

Cohen, D., 1972. Magnetoencephalography: Detection of the brain's electrical activity with a superconducting magnetometer. Science (80-. ). 175, 664-666. https://doi.org/10.1126/science.175.4022.664

Cooper, R., Winter, A.L., Crow, H.J., Walter, W.G., 1965. Comparison of subcortical, cortical and scalp activity using chronically indwelling electrodes in man. Electroencephalogr. Clin. Neurophysiol. https://doi.org/10.1016/0013-4694(65)90088-X

DeLucchi, M.R., Garoutte, B., Aird, R.B., 1962. The scalp as an electroencephalographic averager. Electroencephalogr. Clin. Neurophysiol. https://doi.org/10.1016/0013-4694(62)90028-7

Fuchs, M., Wagner, M., Wischmann, H.A., Köhler, T., Theißen, A., Drenckhahn, R., Buchner, H., 1998. Improving source reconstructions by combining bioelectric and biomagnetic data. Electroencephalogr. Clin. Neurophysiol. 107, 93-111. https://doi.org/10.1016/S0013-4694(98)00046-7

Goldenholz, D.M., Ahlfors, S.P., Hämäläinen, M.S., Sharon, D., Ishitobi, M., Vaina, L.M., Stufflebeam, S.M., 2009. Mapping the signal-to-noise-ratios of cortical sources in magnetoencephalography and electroencephalography. Hum. Brain Mapp. https://doi.org/10.1002/hbm.20571

Hämäläinen, M., Lundqvist, D., 2019. MEG as an Enabling Tool in Neuroscience - Transcending Boundaries with New Analysis Methods and Devices, in: Magnetoencephalography: From Signals to Dynamic Cortical Networks, 2nd Edition. Springer.

Hämäläinen, M.S., Hari, R., IImoniemi, R.J., Knuutila, J., Lounasmaa, O. V., 1993. Magnetoencephalography - theory, instrumentation, and applications to noninvasive studies of the working human brain. Rev. Mod. Phys. 65, 413-497.

Holmes, N., Leggett, J., Boto, E., Roberts, G., Hill, R.M., Tierney, T.M., Shah, V., Barnes, G.R., Brookes, M.J., Bowtell, R., 2018. A bi-planar coil system for nulling background magnetic fields in scalp mounted magnetoencephalography. Neuroimage 181, 760-774. https://doi.org/10.1016/j.neuroimage.2018.07.028

Hoogenboom, N., Schoffelen, J.M., Oostenveld, R., Parkes, L.M., Fries, P., 2006. Localizing human visual gamma-band activity in frequency, time and space. Neuroimage. https://doi.org/10.1016/j.neuroimage.2005.08.043

Huang, M.X., Song, T., Hagler, D.J., Podgorny, I., Jousmaki, V., Cui, L., Gaa, K., Harrington, D.L., Dale, A.M., Lee, R.R., Elman, J., Halgren, E., 2007. A novel integrated MEG and EEG analysis method for dipolar sources. Neuroimage. https://doi.org/10.1016/j.neuroimage.2007.06.002 
livanainen, J., Stenroos, M., Parkkonen, L., 2017. Measuring MEG closer to the brain: Performance of onscalp sensor arrays. Neuroimage 147, 542-553. https://doi.org/10.1016/j.neuroimage.2016.12.048

livanainen, J., Zetter, R., Grön, M., Hakkarainen, K., Parkkonen, L., 2019a. On-scalp MEG system utilizing an actively shielded array of optically-pumped magnetometers. Neuroimage 194, 244-258. https://doi.org/10.1016/j.neuroimage.2019.03.022

livanainen, J., Zetter, R., Parkkonen, L., 2019b. Potential of on-scalp MEG: Robust detection of human visual gamma-band responses. bioRxiv 602342. https://doi.org/10.1101/602342

Jiménez-Martínez, R., Knappe, S., 2017. Microfabricated optically-pumped magnetometers, in: Grosz, A., Haji-Sheikh, M.J., Mukhopadhyay, S.C. (Eds.), Smart Sensors, Measurement and Instrumentation. Springer, Cham, pp. 523-551. https://doi.org/10.1007/978-3-319-34070-8_17

Kamada, K., Sato, D., Ito, Y., Natsukawa, H., Okano, K., Mizutani, N., Kobayashi, T., 2015. Human magnetoencephalogram measurements using newly developed compact module of high-sensitivity atomic magnetometer. Jpn. J. Appl. Phys. 54, 026601. https://doi.org/10.7567/JJAP.54.026601

Kim, K., Begus, S., Xia, H., Lee, S.K., Jazbinsek, V., Trontelj, Z., Romalis, M. V., 2014. Multi-channel atomic magnetometer for magnetoencephalography: A configuration study. Neuroimage 89, 143-151. https://doi.org/10.1016/j.neuroimage.2013.10.040

Knappe, S., Alem, O., Sheng, D., Kitching, J., 2016. Microfabricated Optically-Pumped Magnetometers for Biomagnetic Applications. J. Phys. Conf. Ser. 723, 012055. https://doi.org/10.1088/1742$6596 / 723 / 1 / 012055$

Liu, A.K., Dale, A.M., Belliveau, J.W., 2002. Monte Carlo simulation studies of EEG and MEG localization accuracy. Hum. Brain Mapp. https://doi.org/10.1002/hbm.10024

Molins, A., Stufflebeam, S.M., Brown, E.N., Hämäläinen, M.S., 2008. Quantification of the benefit from integrating MEG and EEG data in minimum $\mathrm{e} 2$-norm estimation. Neuroimage. https://doi.org/10.1016/j.neuroimage.2008.05.064

Mullinger, K.J., Mayhew, S.D., Bagshaw, A.P., Bowtell, R., Francis, S.T., 2013. Poststimulus undershoots in cerebral blood flow and BOLD fMRI responses are modulated by poststimulus neuronal activity. Proc. Natl. Acad. Sci. 110, 13636-13641. https://doi.org/10.1073/pnas.1221287110

Muthukumaraswamy, S.D., 2013. High-frequency brain activity and muscle artifacts in MEG/EEG: a review and recommendations. Front. Hum. Neurosci. 7, 138. https://doi.org/10.3389/fnhum.2013.00138

Osborne, J., Orton, J., Alem, O., Shah, V., 2018. Fully integrated, standalone zero field optically pumped magnetometer for biomagnetism. Steep Dispers. Eng. Opto-Atomic Precis. Metrol. XI 10548.

Pfurtscheller, G., Lopes Da Silva, F.H., 1999. Event-related EEG/MEG synchronization and desynchronization: Basic principles. Clin. Neurophysiol. 110, 1842-1857. https://doi.org/10.1016/S1388-2457(99)00141-8

Sander, T.H., Preusser, J., Mhaskar, R., Kitching, J., Trahms, L., Knappe, S., 2012. Magnetoencephalography with a chip-scale atomic magnetometer. Biomed. Opt. Express 3, 981. https://doi.org/10.1364/BOE.3.000981

Sarvas, J., 1987. Basic mathematical and electromagnetic concepts of the biomagnetic inverse problem. Phys. Med. Biol. 32, 11-22. https://doi.org/10.1088/0031-9155/32/1/004

Shah, V., Knappe, S., Schwindt, P.D.D., Kitching, J., 2007. Subpicotesla atomic magnetometry with a microfabricated vapour cell. Nat. Photonics 1, 649-652. https://doi.org/10.1038/nphoton.2007.201

Sharon, D., Hämäläinen, M.S., Tootell, R.B.H., Halgren, E., Belliveau, J.W., 2007. The advantage of combining MEG and EEG: Comparison to $\mathrm{fMRI}$ in focally stimulated visual cortex. Neuroimage. https://doi.org/10.1016/j.neuroimage.2007.03.066 
Sheng, D., Perry, A.R., Krzyzewski, S.P., Geller, S., Kitching, J., Knappe, S., 2017. A microfabricated opticallypumped magnetic gradiometer. Appl. Phys. Lett. 110. https://doi.org/10.1063/1.4974349

Tierney, T.M., Holmes, N., Mellor, S., López, J.D., Roberts, G., Hill, R.M., Boto, E., Leggett, J., Shah, V., Brookes, M.J., Bowtell, R., Barnes, G.R., 2019. Optically pumped magnetometers: From quantum origins to multi-channel magnetoencephalography. Neuroimage In Press.

https://doi.org/10.1016/j.neuroimage.2019.05.063

Tierney, T.M., Holmes, N., Meyer, S.S., Boto, E., Roberts, G., Leggett, J., Buck, S., Duque-Muñoz, L., Litvak, V., Bestmann, S., Baldeweg, T., Bowtell, R., Brookes, M.J., Barnes, G.R., 2018. Cognitive neuroscience using wearable magnetometer arrays: Non-invasive assessment of language function. Neuroimage 181, 513-520. https://doi.org/10.1016/j.neuroimage.2018.07.035

Whitham, E.M., Pope, K.J., Fitzgibbon, S.P., Lewis, T., Clark, C.R., Loveless, S., Broberg, M., Wallace, A., DeLosAngeles, D., Lillie, P., Hardy, A., Fronsko, R., Pulbrook, A., Willoughby, J.O., 2007. Scalp electrical recording during paralysis: Quantitative evidence that EEG frequencies above $20 \mathrm{~Hz}$ are contaminated by EMG. Clin. Neurophysiol. 118, 1877-1888. https://doi.org/10.1016/j.clinph.2007.04.027

Zhang, Z., 1995. A fast method to compute surface potentials generated by dipoles within multilayer anisotropic spheres. Phys. Med. Biol. 40, 335-349. https://doi.org/10.1088/0031-9155/40/3/001

Zimmermann, R., Scharein, E., 2004. MEG and EEG show different sensitivity to myogenic artifacts. Neurol. Clin. Neurophysiol. NCN 2004, 78. 